\title{
STEMXEL: INTERACTIVE STRUCTURE FOR ADAPTIVE DESIGN
}

\author{
PIER ALESSIO RIZZARDI ${ }^{1,2,3}$, BASAK SAKCAK ${ }^{1}$, HANKUN ZHANG $^{1}$, EDOARDO GIANCOLA $^{1,4}$, \\ MARTIN HUBA $^{1} \&$ ALEJANDRO REY YAMHURE ${ }^{1}$ \\ ${ }^{1}$ TCA Think Tank. \\ ${ }^{2}$ STUDIO Architecture and Urbanism Magazine. \\ ${ }^{3}$ l'ARCA International. \\ ${ }^{4}$ Zarcola Studio.
}

\begin{abstract}
StemXel is a response to the need of continuous change of functions in interior design and architecture. It can be converted into mutable forms with the use of a permanent material without any waste and provide physical change with seamless work. The structure is flexible to turn a simple single space into an adaptable environment that can be used for multiple programs at multiple instances. The structure is designed to be implemented as a furniture, for housing, commercial activities and for spaces that are undergoing a constant change. StemXel responds to different inputs, digital or analogic, in order to create a fast and fluid physical change. Its flexibility allows the possibility to absorb digital information, meanwhile reflecting it in a tangible form. It is a structure that goes beyond two-dimension, yet involves the totality of a defined volume. Intelligent interaction between users and space is one of the major characteristics to be exploited in future design development.

Keywords: furniture, interaction, programmability.
\end{abstract}

\section{INTRODUCTION}

Considering the state of the technology and hyper-connectivity provided by mass media of the virtual world, we are facing numerous possibilities given by the contemporary life and the objects around us. It is the consequence of this period of time, pushing us to relate to the world in unexpected ways: interaction with objects, self-generating and co-sharing information platform. So far the field of interactive design is still at its initial phase, yet we can already see examples that react to light, heat, noise, pressure, and various human gestures and activities. Our objectives considered as daily necessities are simply the projection of a need in a given moment in time, however the spaces around us today are constructed to be permanent, but only to fulfil momentous requirements. Proposed StemXel in this context is an extension of our daily needs, building up a framework that changes its form to meet user requirements at the specific moment. It is an installation that preserves the same material while changing the functionality. Hence, it is sustainable both in terms of material and space. It is a grid structure composed of independent mobile beams, which function individually to assemble as a consequence of either pre-designed, or immediate and spontaneous intentions, as a direct reaction to the latest experimentations in interactive design. One recent example is for a shape display, inFORM [1]. This work improves the traditional shape displays that focus mainly on the graphical aspects to a new level of tangible interaction. Interactive Pixel Wall [2] is an installation that obtains user input to deform an organic shape preliminarily set by the designer, while constructing a desired piece of furniture. Jonpasang group [3] utilizes 3D pixels to obtain kinetic landscape in 2012 Yeosu EXPO site. More inspirations were drawn from artistic examples in an interactive manner with the users such as PomPom Mirror [4] and Kinetic Sculpture [5]. In another project, the Translated Geometries, the programmability of materials are developed in an intellectual way [6]. StemXel employs these 
working principles, engaging in an interactive user experience and holding for the duration of time as needed. Therefore, the objects can be created and recreated depending on the user requirements.

\section{DESIGN CONCEPT}

StemXel is a transformable furniture installation, composed by individually motivated pieces, thus allowing infinite possibilities to serve the needs of each occasion and event accordingly. When StemXel is treated as interior furniture, it is a vertical structure that can transform into shelves, seats, armchairs, tables and stools. Depending on functional purposes, users input different information as needed, every element altogether can shape a set of furniture (Fig. 1). By adopting different applications, it realizes 3D models and images, dynamically presenting a much stronger sense that can be perceived directly.

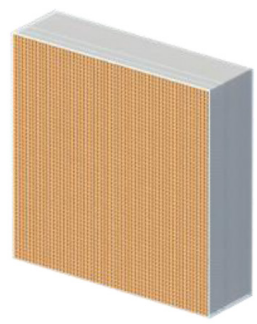

(a) StemXel at its initial position

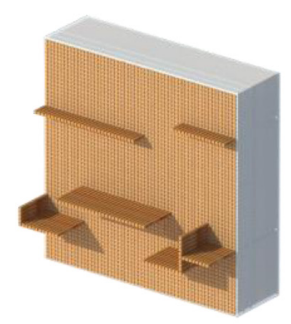

(b) StemXel at its final position

Figure 1: Starting from the initial position, StemXel can be extruded to obtain a desired final shape, could it be a shelf, table, chair etc.

\section{TECHNICAL SPECIFICATION}

\subsection{Mechanism}

StemXel structure contains a matrix of beams with a dimension of $2.5 \times 2.5 \times 80 \mathrm{~cm}$, individually connected to motors controlled by a micro-controller. The operation of StemXel structure will have two modes, the first mode is where the structure moves to form the desired shape, and the second mode allows interaction with the user by means of sensors. Each beam is installed in a metal profile, which serves both to guide the extruding direction and as a supporting structure. The extrusion of each beam is obtained by the help of a stepper motor connected to a lead screw assembly, a mechanism that converts the rotational motion into linear movement, allowing each beam to slide in and out of the profile (Fig. 2). Due to the fact that the beam size is kept small allowing the required motion to be obtained with a small amount of energy, a small stepper motor is selected for its size.

Beams are inserted in metal profiles to compose a supporting structure, where each metal profile is stashed on one another to create the entire wall. These profiles are held together by a double metal beams positioned at the bottom as a base and on top, tied by metal tie rods to prevent movement and deformation of the combined structure (Fig. 3). 


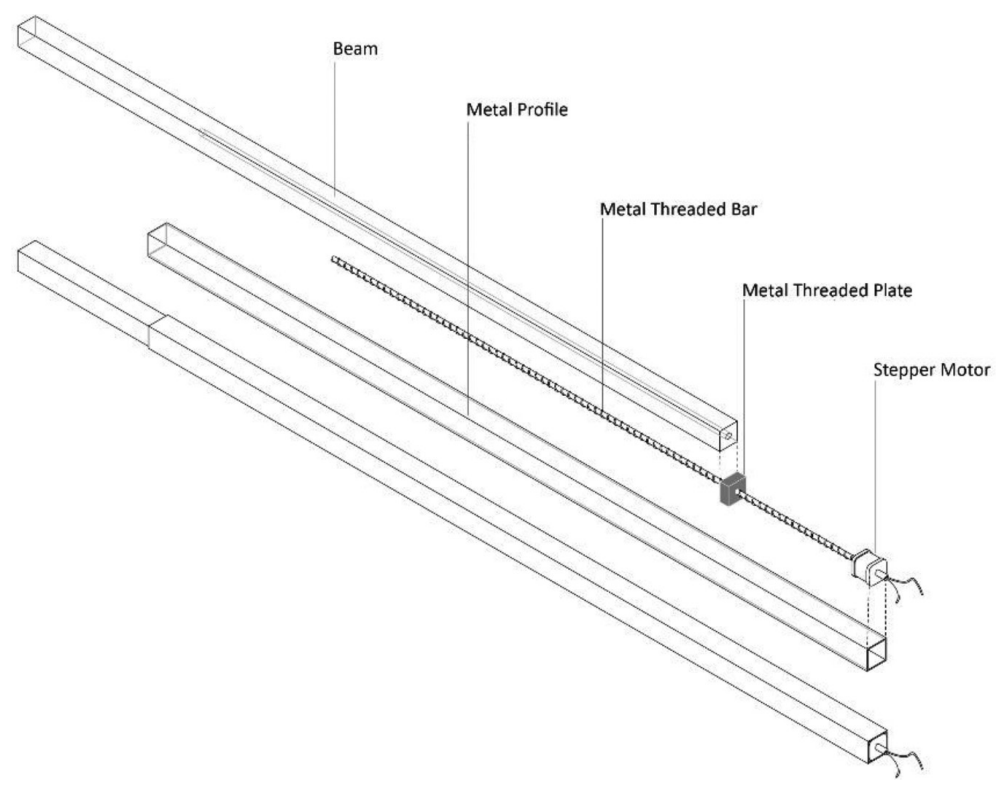

Figure 2: Extrusion mechanism of one beam.

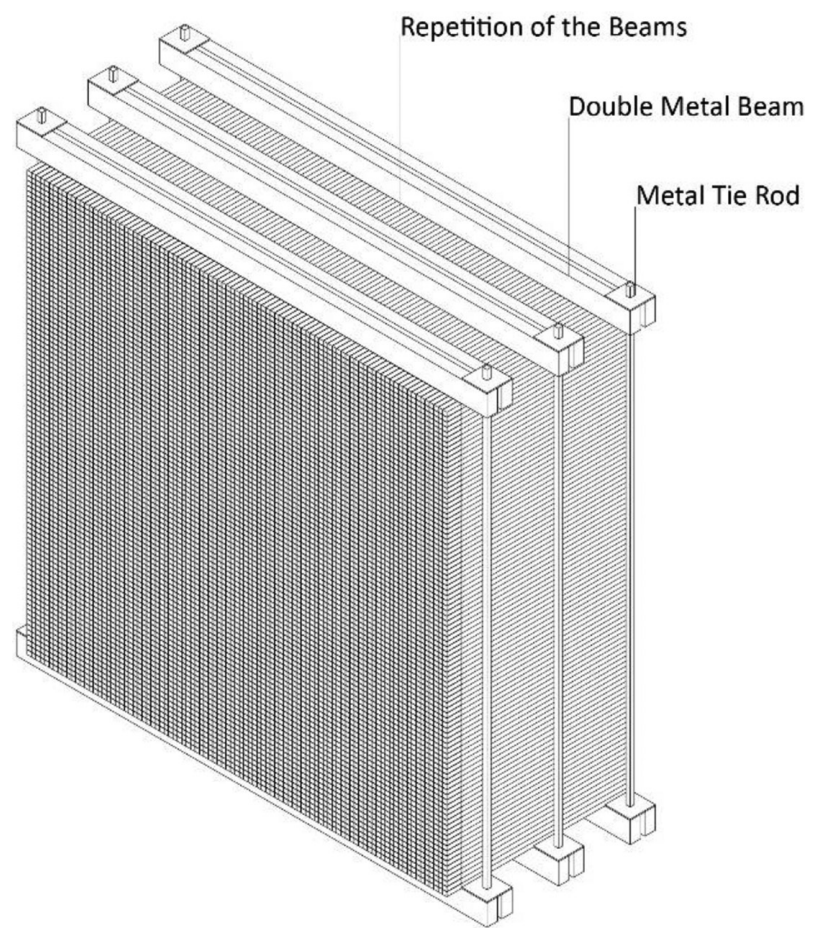

Figure 3: Supporting structure composed of metal profiles tied together. 


\subsection{Material}

In order to have a structure able to sustain the weight of objects and people, we investigate the resistance of the beams. For that purpose we assume a concentrated load at the end of the beam at its full extruded state and compute the maximum load $P_{\max }$ that the beam can sustain for different materials using

$$
P_{\max }=\frac{\sigma \times \frac{B \times H^{2}}{6}}{b}
$$

where $\mathrm{B}$ and $\mathrm{H}$ are the width and the height of the beam cross-section correspondingly, $\sigma$ is the bending resistance and finally $b$ is the maximum extrusion state of $600 \mathrm{~mm}$,

Supposing that the interaction between the weight of the human body and the structure will not have only one point on one single beam, we assume that the minimum number of beams to partially sustain the body will be 5 beams, which means in order for the structure to resist to the body weight, we have to divide the maximum load applicable by 5 times. Assuming a maximum body weight of $150 \mathrm{~kg}, 50 \%$ of the actual weight will function on the structure, which means:

$$
P=\frac{\frac{150}{2}}{5}=15 \mathrm{Kg}
$$

which means $15 \mathrm{~kg}$ on each beam. We consider the actual supporting area for the maximum weight of $150 \mathrm{Kg}$ will cover a minimum of 15 beams, which means $10 \mathrm{~kg}$ on each beam. Considering this hypothesis and taking into account the maximum loads computed and provided in Table 1, we arrive at the conclusion that it is more secure to use a high Spruce Wood or Corian material, which means marble is not applicable in this case.

\subsection{Actuation of the beams}

In order to form a desired object on StemXel or create a surface using the beams, each component has to receive the information about for how long it should be extruded. For that purpose, we developed a simple algorithm that automatically decides which beams should be activated and for how long. The first part of the algorithm relates the information obtained from the user and the model to the StemXel surface by computing the exact extrusion distance for each beam to be activated. Following that the second part generates the corresponding information to be sent to the motors.

Table 1: Materials with different bending resistances and the corresponding maximum loads that the beams can sustain.

\begin{tabular}{llll}
\hline & Carrara Marble & Spruce Wood & Corian \\
\hline$\sigma$ & $20.3 \mathrm{MPa}$ & $100 \mathrm{MPa}$ & $67 \mathrm{Mpa}$ \\
$P_{\max }$ & $88.1 \mathrm{~N}(8.98 \mathrm{~kg})$ & $434 \mathrm{~N}(44.29 \mathrm{~kg})$ & $291 \mathrm{~N}(29.69 \mathrm{~kg})$ \\
\hline
\end{tabular}


We define the surface that corresponds to the arrangement of the beams when they are all in their initial positions as the "base surface". The algorithm relies on the discretized representation of this base surface; such that the total surface is defined as a grid with a cell size equal to the beam cross-section. The algorithm then, decides on the beams to be activated and the extrusion distance from their initial positions. The functionality of the algorithm is as follows:

- Obtaining user input: In this step user is asked to select a model to realize and place it on StemXel using a user interface.

- Highlighted Beams: In this step the algorithm obtains the projection of the model on the base surface. Following that, all the cells that contain a piece of the projected area is assigned as highlighted cells, hence beams.

- Extrusion Distance: In order to compute the distance that each beam should be extruded from the initial surface, the model is intersected using a ray that starts from the centroid of each cell and normal to the base surface. The length $d_{z}$ of the line segment that is defined by the centroid of each grid cell and the intersection point is the exact length of the beam to be extruded (Fig. 4).

The second part of the actuation algorithm translates the information about the beams to be activated and their extrusion distance into a binary signal used to control the motors in order to get the desired shape. Since stepper motors are used, each pulse sent to them represent a fixed angle of rotation that converts into a fixed translation. This distance is easily calculable from the motor specifications. Therefore, it is possible to translate the desired extension of each beam from the model into a number of pulses that need to be fed to the motor in order to make the pixel move to the desired position. On the other hand, due to the discretized nature of the motion the exact distance from the base surface to the final distance computed is obtained up to a precision. After calculating how many pulses must be sent to each pixel, the software generates a series of binary numbers containing the information regarding which motors must be activated. The data are then transferred to the microcontroller that will send

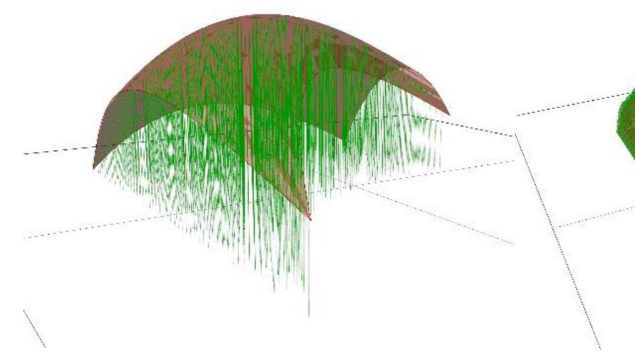

(a)

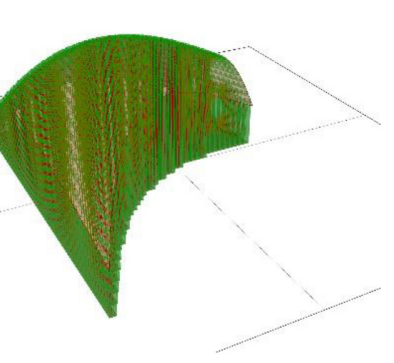

(b)

Figure 4: Grasshopper simulations for the realization of a parametric surface. (a) The rays originated from the centroids of the highlighted beams and normal to the base surface are intersected with the surface. Length of each line segment shown with green, gives out the extrusion distance for each of the corresponding beams. (b) Highlighted beams are extruded up to the surface given by the user. 
out the signals to the system, using a network of transfer registers, which store the information temporarily until the signal has completely propagated though the grid. After the signal has reached the final part of the grid, a trigger signal will be sent so that the desired driver circuits activate the stepper motors. The second mode of operation, defined as the interactive mode, is based on sensors. The sensors, all connected through a network of transfer registers, when activated, send a signal that is received by the microcontroller. The information received is then processed (either directly by the microcontroller or by a PC) and transformed into a series of binary signals that will control the motors. This signal is then transferred through the system to activate the desired motors.

\section{SIMULATIONS}

We used the Grasshopper environment to verify the first part of the algorithm. For that purpose, we have developed three different simulation environments, in the first case StemXel is used to obtain a parametric surface In this case the StemXel is programmed to adopt the shape of an organic surface (Fig. 5). In the second case realization of furnitures is exploited. We have used the models of chairs designed by Caroline Schlyter together with the wiggle side chair designed by Frank Gehry and Zig Zag chair designed by Gerrit Rietveld after placing them on the StemXel base surface (Fig. 6). The third environment is used to test the possible user interaction. For that purpose, we have defined a point to generate an attractive field such that the collective appearance of the beams would be directed towards this point (Fig. 7). The distance between the centre of beams and the point gives the final length of the extrusion. Design configuration has the potential to be additionally developed: taking into consideration the other environmental inputs such as sound, light, movements and define them as potential attractors.

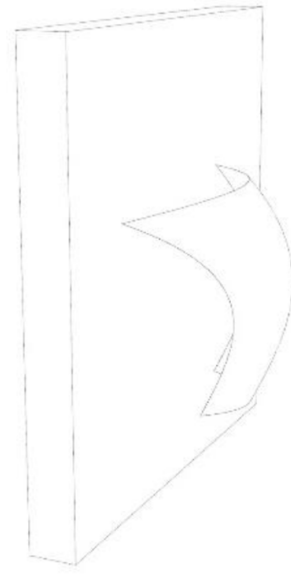

(a)

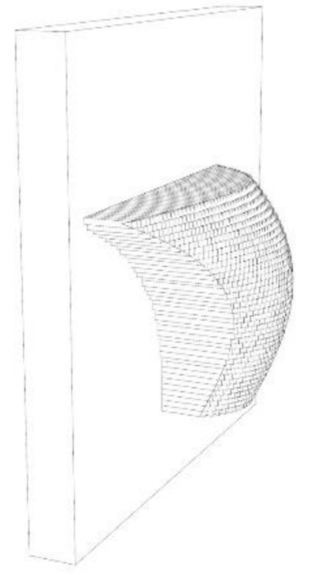

(b)

Figure 5: Simulations using Grasshopper for realizing a surface. (a) The user inputs the given shape to be realized and the location of it with respect to the base surface. (b) The algorithm automatically computes the beams to be activated and moves them such that they form the desired shape. 


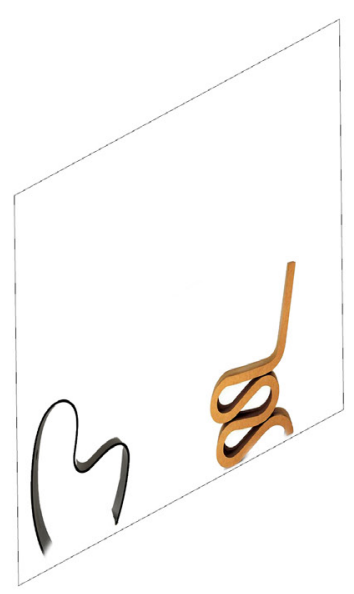

(a)

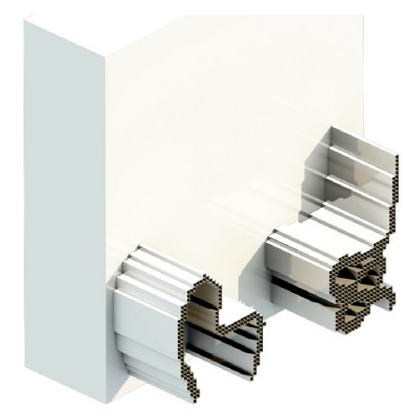

(b)

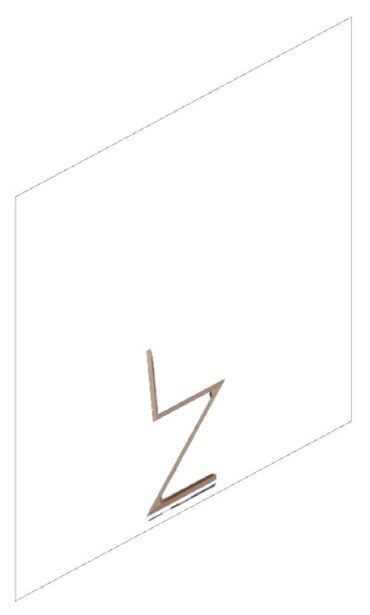

(c) (d)

Figure 6: Simulations using Grasshopper for realizing furniture. The user inputs the 3D models of the objects to be realized and locates them on the base frame. The algorithm automatically computes the beams to be activated and extrudes them up to the maximum distance to the base surface regarding the model. (a) Chair designed by Caroline Schlyter together with the wiggle side chair designed by Frank Gehry (c) Zig Zag chair designed by Gerrit Rietveld. (b, d) Chairs realized using StemXel in Grasshopper simulation. 


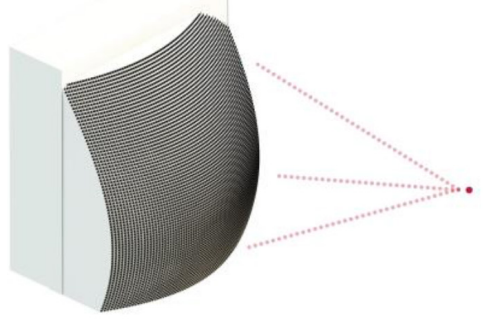

(a)

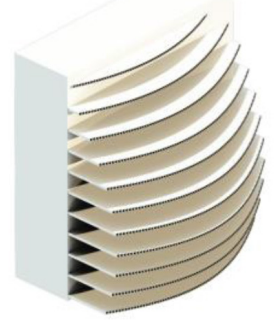

(b)

Figure 7: StemXel various testing positions: Design test with an attractor. The basic concept consists in assigning the relationship between the object (beams) and the certain points in space (attractor points), to parameters of the geometric manipulations to objects.

\section{APPLICATIONS}

- Configured by hand, pushing and pulling parts of the structure giving freedom to the practical experimentation (Fig. 8).

- Configured through a device choosing from different types already designed and tested by the designers (Fig. 9).

- Configured by taking a picture to what you like and let the structure reproduce it automatically (Fig. 10).
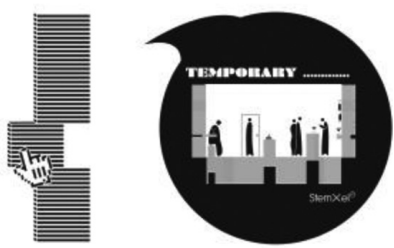

Figure 8: Direct action of the user to shape the space around him.

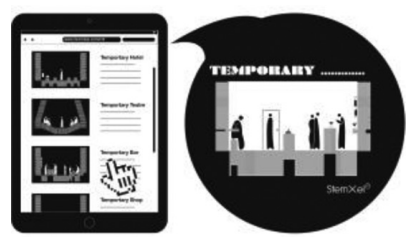

Figure 9: Choosing the shape according to the predefined layout.
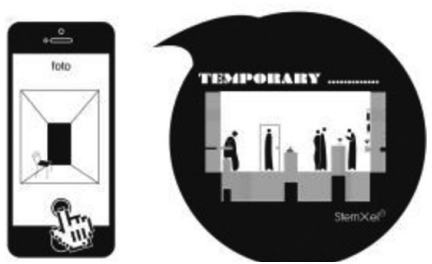

Figure 10: Scanning objects and environment to reproduce it on the structure. 


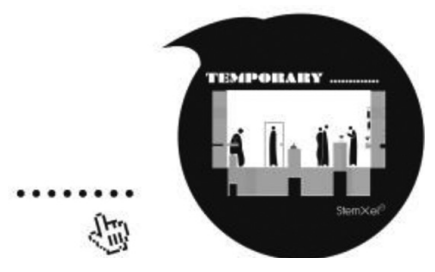

Figure 11: The structure can learn the user routine and interpret the space required.

- Configured in many other ways in the future of technology giving the possibilities to let your imagination be alive and be freedom (Fig. 11).

\section{CONCLUSION}

StemXel is the direct response to the need of our ever-changing living environment. Its possibilities range from the simple manual formation to a more complex pre-designed digital input which allows the mind to imagine its numerous variations.

For the future works, we are going to build a prototype with a small subset of beams and verify the functioning of the proposed methods with experiments using the straightforward functioning only. After this part is completed, a more complicated functioning, the interactive mode, will be tackled. We expect this part to be technically more challenging due to the usage of sensors.

\section{REFERENCES}

[1] Follmer, S., Leithinger, D., Olwal, A., Hogge, A. \& Ishii, H., inFORM: dynamic physical affordances and constraints through shape and object actuation. In UIST, 13, pp. 417-426, 2013.

[2] Mahli, A.M., Massani, S.A., Sroor, N., Interactive Pixel Wall. [Installation]. Istanbul, Turkey: Macka Park, 2015.

[3] Mok, J., 3D Hyper-Matrix. [Installation] Yeusu, South Korea: Hyundai Motor Group Pavilion, YEOSU EXPO, 2012.

[4] Daniel Rozin, PomPom Mirror, 2015.

[5] ART+COM Studios, Kinetic Sculpture - The Shapes of Things to Come, BMW Museum, Munich, Germany, 2008.

[6] Translated Geometries: Master in Advanced Architecture project, developed by students: Efilena Baseta, Ece Tankal, Ramin Shambayati; Senior Faculty: Areti Markopoulou; Faculty Assistants: Alexandre Dubor \& Moritz Begle; Research Line: Digital Matter Intelligent Constructions, 2013/14. 